\title{
Numerical simulation of undrained cyclic behavior for desaturated silica sands
}

\author{
Kengo Kato ${ }^{\text {i) }}$, Koichi Nagao ${ }^{\text {ii) }}$, and Naoaki Suemasa ${ }^{\text {iii) }}$ \\ i) Ph.D, Geotechnical Research Engineer, Sato Kogyo, 14-11 Morinosato Aoyama, Atsugi, Kanagawa, Japan. \\ ii) Geotechnical Research Engineer, Sato Kogyo, 14-11 Morinosato Aoyama, Atsugi, Kanagawa, Japan. \\ iii) Professor, Tokyo City University, 1-28-1 Tamazutsumi Setagaya, Tokyo, Japan.
}

\begin{abstract}
Desaturation is an effective ground improvement for mitigating liquefaction-induced ground failures. This paper aims to discuss numerical capabilities for simulating the undrained cyclic behavior of desaturated silica sands. The laboratory experiments were initially conducted to investigate desaturation effects on medium dense desaturated silica sand behavior $\left(\mathrm{D}_{\mathrm{r}}=60-65 \%, \mathrm{~S}_{\mathrm{r}}=91-100 \%\right)$ under undrained triaxial loading conditions. Desaturation was achieved by injecting micro-bubbled water. The experimental results showed an increase of the angle of a phase transformation line, $\phi_{p l}$, in $4-5^{\circ}$, no desaturation effects on the angle of a critical state line, $\phi_{c l}$, and less degradation of Young modulus comparing to the saturated condition. The numerical analysis was secondarily performed to simulate the experimental results using LIQCA2D17. The comparisons to the preliminary experimental result showed that failure criteria were critical on stress-strain behaviors, and the Yasufuku criterion was suitable for both saturated and desaturated conditions. The desaturation effects were further discussed. The desaturation critically affected the stress-strain behavior after the stress path reached the phase transformation. The desaturated silica sands exhibited continuous modulus reduction although the specimens were fully liquefied (i.e., EPWP ratio nearly equaled to 1.0). Liquefaction of those desaturated silica sands was characterized as "slowly flow deformation."
\end{abstract}

Keywords: Desaturation, Liquefaction, Numerical simulation, Ground improvement, Unsaturated soil mechanics

\section{INTRODUCTION}

Liquefaction-induced geohazards have captured great attention since the 1964 Alaska earthquake and the 1964 Niigata earthquakes induced devastating damages on slopes, bridges, building foundations, and buried structures. Many ground improvement technique for mitigating liquefaction-induced ground failures has been developed as improving the understanding of liquefaction mechanisms. The generation of excess pore water pressure under undrained loading condition is a key feature of liquefaction phenomena. Desaturation methods have been used as an effective ground improvement for liquefaction-induced geohazard mitigation.

Desaturation (or a degree of saturation, $S_{r}$ ) effects under undrained cyclic loading have been mainly investigated in elemental test scale or model test scale. Triaxial and hollow cylinder test devices are a commonly used one to examine the undrained cyclic behavior of sands in element test scale. The relationships between cyclic stress ratio, CSR, and a number of stress cycles, $N_{c y c}$, are usually used to characterize liquefaction resistances with cyclic strain amplitude. Several studies showed that desaturated sands have higher liquefaction resistance than saturated sands (e.g., Yoshimi et al. 1989, Ishihara et al. 1998, Okamura and Soga 2006). Marasini and Okamura (2015) showed that desaturation reduced excess pore water pressure (EPWP) development and settlements of the container sands in centrifuge tests. These experimental results clearly show that a degree of saturation, $S_{r}$, significantly influences liquefaction characteristics of sands under undrained cyclic loading. Specifically, excess pore water pressure responses of desaturated sands explicitly differ from saturated sands. However, current recommended liquefaction potential evaluation procedures, such as Boulanger and Idriss (2014), do not take into account for $S_{r}$ effects on liquefaction resistance. Therefore, there is a need for a more rigorous analysis for desaturated sands subjected to undrained cyclic loading.

The objective is to discuss numerical capabilities for simulating the undrained cyclic behavior of desaturated silica sands. Firstly, the undrained triaxial cyclic loading tests (CTX) are conducted on both saturated and desaturated conditions. Desaturation effects are evaluated comparing stress-strain relationships, strain-pore water pressure responses, excess pore water pressure developments, stress paths, and liquefaction resistance curves (i.e., $C S R-N_{c y c}$ relationships). 
Secondary, the numerical analysis is performed to simulate the experimental results using LIQCA2D17. The desaturation effects are further discussed to simulate strain behavior after stress path reaches phase transformation lines. The numerical simulation indicated that volumetric characteristics are important to desaturated silica sand behavior for liquefaction resistance.

\section{CYCLIC TRIAXIAL EXPERIMENTS}

\subsection{Specimen preparation and Test procedures}

Azegami (2008) conducted undrained cyclic shear tests using triaxial cell devices for saturated and desaturated silica sands. A brief of test procedures was described herein. Silica sand properties used for the experiment were shown in Table 1. Maximum and minimum void ratio, $e_{\max }$, and $e_{\min }$, were determined following JIS A 1224, specific gravity of sand particles, $G_{s}$, were determined following JIS A 1202, and coefficient uniformity $U_{c}$ and curvature $C_{c}$ were determined following JIS A 1204. No fine particles were contained, then the silica sand was classified as poorly graded clean sand (SP) based on the unified soil classification system.

Specimen preparations were followed JGS 0520. Sand specimens, which had a $50 \mathrm{~mm}$ diameter and a $100 \mathrm{~mm}$ height, were prepared by pluviating dry sands into the mold to a relative density, $D_{r}$, of approximately $60 \%$. The specimen was covered with a 0.5 -mm-thick latex membrane using a membrane stretcher, sealed to the pedestal and the cap with O-rings. Small vacuum stress was applied to confine specimens during setup of the triaxial cell. After application of the vacuum, the specimen height was measured, and the diameter at the top, center, and bottom of the specimen was also measured to calculate average volume.

Fully saturated specimens were firstly prepared with a back pressure of $101 \mathrm{kPa}$. Specimen saturation with de-aired water was performed, and the specimen height was re-measure using an LVDT strain gauge. Once B-value $>0.95$ was confirmed, the back pressure was reduced to $0.01 \mathrm{kPa}$ to inject micro-bubbled water, which contains tiny air bubble of a diameter of $10-100 \mu \mathrm{m}$, into the saturated specimen. During the injection, air volume contained within the specimen was monitored to estimate a degree of saturation, $S_{r}$. Each specimen was consolidated under isotropic confining stress of $98 \mathrm{kPa}$. $S_{r}$ was measured under a back pressure of $98 \mathrm{kPa}$. During the consolidation process, ejected pore water volume and a new height were measured, then a new $D_{r}$ of the consolidated specimen was calculated before applying cyclic loading.

A series of cyclic triaxial experiments (CTX) was performed on both saturated and desaturated sands following the methodology described in JGS 0541. All CTX tests were conducted by applying stress controlled
Table 1. Silica sand properties

\begin{tabular}{cc}
$e_{\min }$ & 0.961 \\
$e_{\max }$ & 0.603 \\
Coefficient of Uniformity, $U_{c}$ & 1.28 \\
Coefficient of Curvature, $C_{c}$ & 1.06 \\
Specific Gravity, $G_{s}$ & 2.64 \\
\hline
\end{tabular}

sinusoidal cyclic loading at a frequency of $0.08-0.2 \mathrm{~Hz}$ under undrained condition. Stress amplitude was characterized in terms of cyclic stress ratio (CSR), which is a ratio of a single amplitude of deviator stress, $\sigma_{d},\left(=\sigma_{a}-\sigma_{c}\right.$, where $\sigma_{a}$ is axial stress and $\sigma_{c}$ confining stress) to the initial effective confining stress, $\sigma^{\prime}{ }_{\mathrm{c} 0}$ in the triaxial stress condition. CSRs in the range of 0.15-0.20 and $0.25-0.30$ were applied on the saturated and desaturated silica sand until double amplitude axial strains (DA) in excess of 5\% was achieved.

\subsection{Experimental results and Discussions}

Fig.1 compared representative results of the isotopically consolidated saturated and desaturated silica sand. The comparisons of the effective stress path and the excess pore water pressure (EPWP) development provided evidence of limitations for EPWP ratio-based liquefaction criterion. For the saturated condition, the EPWP ratio reached over 0.95 in two cycles after the stress path reached phase transformation, and the axial strain, $\varepsilon_{a}$, rapidly increased in extension. Then, both EPWP and double amplitude axial strain (DA) based criterion (say, $\mathrm{DA}=2.5 \%$ ) provided the same number of stress cycles, $N_{c y c}$. That is usually observed for poorly graded sands in the triaxial stress condition. For the desaturated condition, it could be seen that the $\varepsilon_{a}$ apparently increased in the EPWP ratio around 0.4 and continuously developed in extension. The maximum EPWP ratio was still nearly 0.8 although the DA reached $2.5 \%$. The EPWP value was smaller than the saturated condition although the strain level was the same. The result showed that the differences between the EPWP-based criterion and the strain-based criterion become significant for the desaturated condition. The strain-based criterion for the onset of liquefaction was used for both saturated and desaturated condition because a different range of the frequency was employed. The stress path in p'-q space showed that the saturated silica sand immediately generated the EPWP in the first cycle in compression, and the mean effective stress decreased with an increase of the $N_{c y c}$. The desaturated silica sands exhibited dull EPWP and axial strain developments. Additionally, dilative responses were observed when the $\varepsilon_{a}$ was over $4 \%$ in extension. That showed that there was a phase transformation state as same as the saturation condition.

Fig.2 showed the stress-strain relationships and the $N_{c y c}$ required to reach the DA in $2.5 \%$ and $5.0 \%$. It could be seen for the desaturated condition that the larger strains developed in extension, the loops moved 

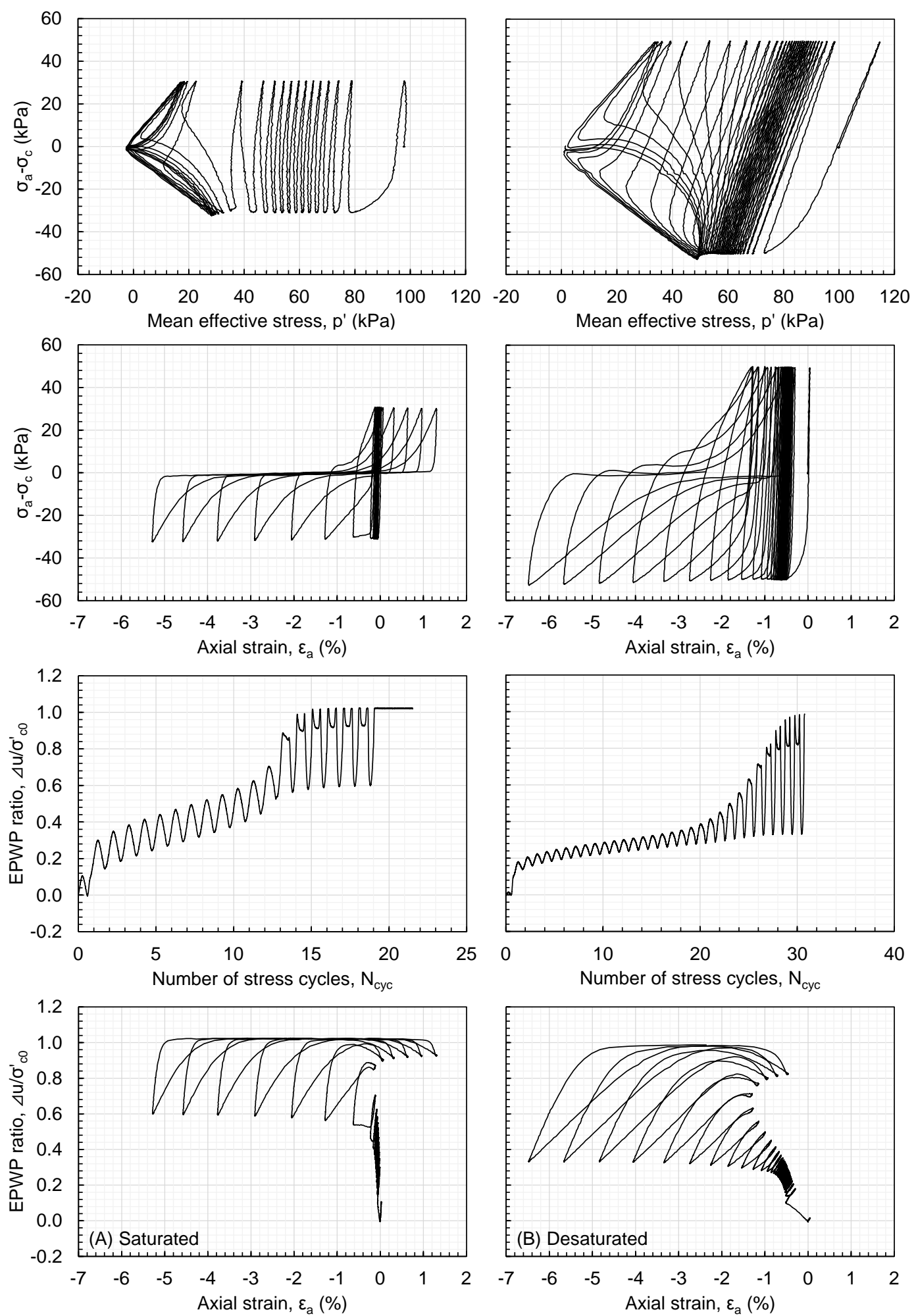

Fig. 1. The comparisons of undrained cyclic behavior for (A) saturated conditions and (B) desaturated conditions. (Data from Azegami 2008)

in extension, the area of the loops expanded at the cycle 27; however, the shape of the loop became similar to the saturated one at the cycle 30 . The desaturated specimen underwent dilation during shearing, leading to the development of "banana loops" to "S-shape loops." That showed that young modulus reductions with the strain development more slowly decreased than the saturated condition. Fig. 3 showed the angle of a phase transformation line, $\phi_{p l}$, and a critical state line, $\phi_{c l}$, in compression and extension with $S_{r}$. Fig.3 presented that the $\phi_{p l}$ and the $\phi_{c l}$ in compression fell within the same range regardless of $S_{r}$. For example, the 

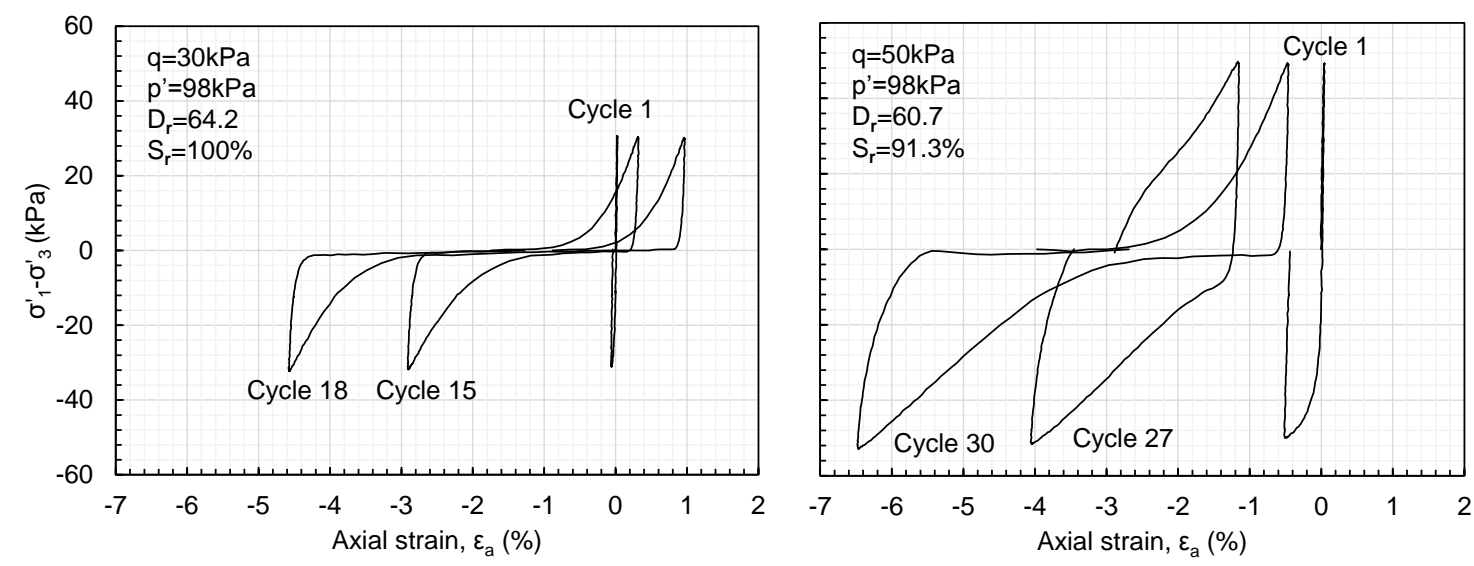

Fig. 2. Stress-strain relationship for the saturated and desaturated sands.
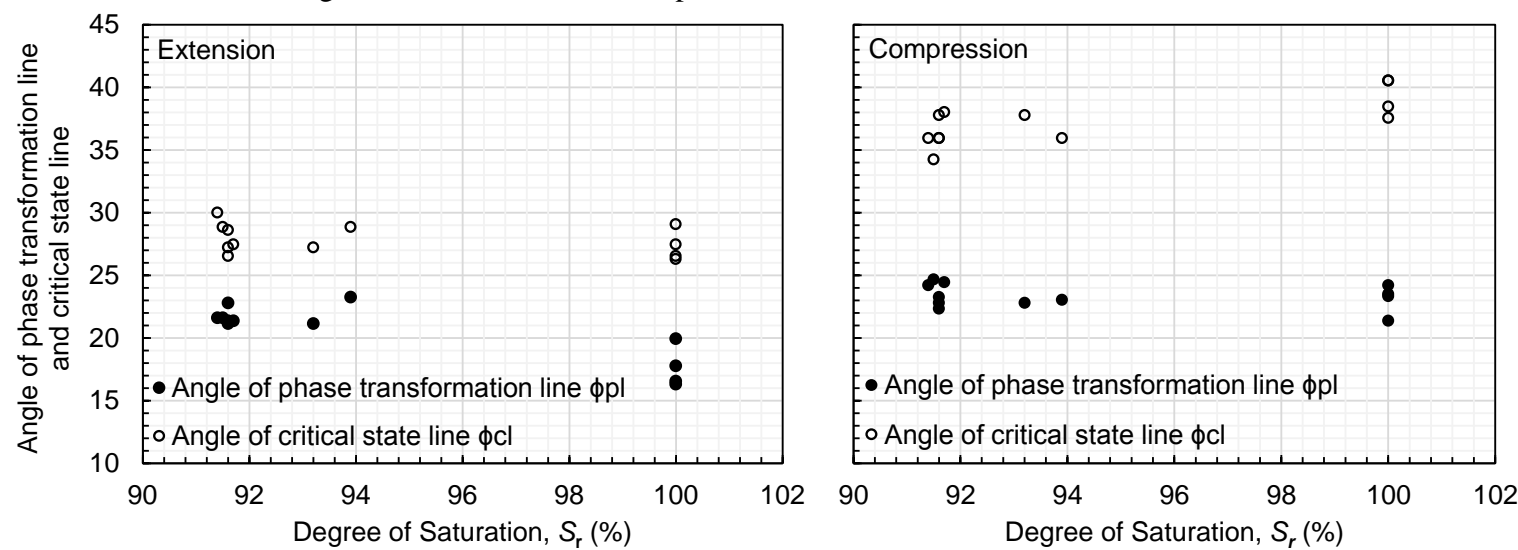

Fig. 3. Angle of the pahse transformation line and the ctirical state line.

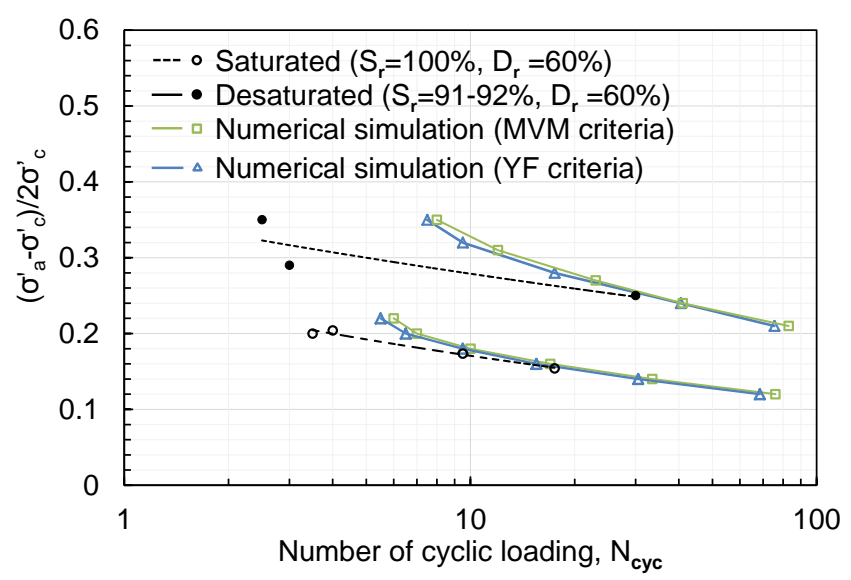

Fig. 4. Liquefaction resistance curves of the experimental and simulation results.

range of the $\phi_{p l}$ was $22-25^{\circ}$ for $S_{r}=91-94 \%$ and $21-24^{\circ}$ for $S_{r}=100 \%$. The range of the $\phi_{c l}$ was $34-38^{\circ}$ and $37^{\circ}$ $40^{\circ}$ for those $S_{r}$, respectively. However, the tendency was slightly different in extension. The range of $\phi_{p l}$ was 21-24 and $16-20^{\circ}$ for the desaturated and saturated condition, respectively, however, the $\phi_{c l}$ fell within the range of $26-30^{\circ}$ for both conditions. There was a difference in $4-6^{\circ}$ for the $\phi_{p l}$, although the $\phi_{c l}$ was almost the same in extension. Therefore, the results showed that a degree of saturation at the $S_{r} \approx 91-100 \%$ did not influence the $\phi_{c l}$ in compression and extension, however slightly increased the $\phi_{p l}$ in extension. So, only the angle of a critical state line was unique at those degrees of saturation ranges.

Fig.4 showed liquefaction resistance curves for both saturated and desaturated condition. All specimens were loaded to a maximum peak deviator stress of $30-60 \mathrm{kPa} \quad(\mathrm{CSR}=0.15-0.20)$ and $50-70 \mathrm{kPa}$ $(\mathrm{CSR}=0.25-0.30)$ until the DA excessed in 5\%. The results showed that the $N_{c y c}$ with the CSR of 0.20 was 3 for the saturated condition, and that number with the CSR of 0.25 was 30 for the desaturated condition. Those results presented that desaturation increased the liquefaction resistance. That is fairly consistent with Yoshimi et al. (1989), Tsukamoto et al. (2002), and Okamura and Soga (2006). 

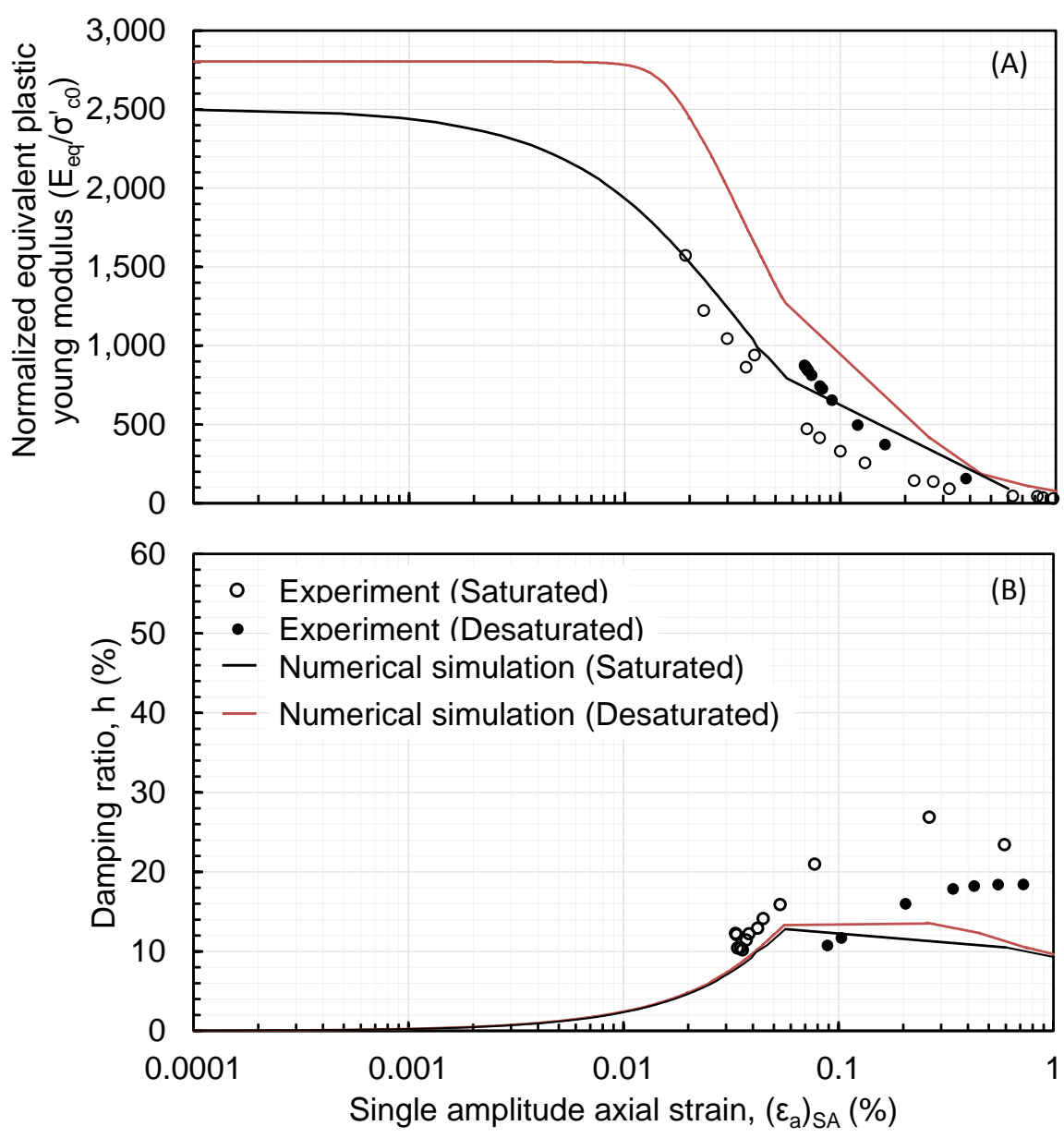

Fig. 5. Modulus reduction behavior and hysteresis damping of the experimental results and the numerical simulations.

Fig.5 (A) and (B) showed modulus reduction and damping ratio, $h$, behavior with axial strain developments. The estimation of equivalent young moduli, $E_{e q}$, and damping ratio, $h$, was followed JGS 0542. Fig.5 (A) showed that normalized equivalent young moduli, $E_{e q} / \sigma^{\prime}{ }_{c 0}$, decreased with an increase of the $\varepsilon_{a}$ for both conditions. The $E_{e q} / \sigma^{\prime} 0$ of the saturated condition was 415 at the $\varepsilon_{a}$ of $0.08 \%$, and the one of the desaturated condition was 587 at the $\varepsilon_{a}$ of $0.09 \%$. Then, the results showed that the desaturation condition exhibited high young modulus at the same strain level. Additionally, the damping ratio, $h$, increased with an increase of the $\varepsilon_{a}$. The peak damping ratio of the saturated condition was $27 \%$ at the $\varepsilon_{a}$ of $0.27 \%$, and the one of the desaturated condition was $17.5 \%$ at the $\varepsilon_{a}$ of $0.38 \%$. The desaturated condition exhibited a smaller damping ratio. Therefore, the experimental results showed that $S_{r}$ also influenced the modulus reduction behavior and the damping ratio.

The experimental results are summarized below.

1) The angle of a critical state line, $\phi_{c l}$, is the same for the range of $S_{r}=91-100 \%$ in extension; however, Desaturation increased the angle of a phase transformation line, $\phi_{p l}$, in $4-6^{\circ}$ comparing the saturated condition. Those angles are mostly the same regardless of $S_{r}$ in compression.
2) The modulus reduction and damping ration behavior for the desaturated condition highly depend on axial strain development as same as the saturated condition. The young modulus reduction decreases with a decrease of $S_{r}$, and the damping ratio decreased with a decrease of $S_{r}$ as well.

3) The shape of the stress loop transitions from "banana loops" to "S-shape loops" with an increase of axial strain for the desaturated condition. Stiffness evolution dramatically changes and highly depends on the axial strain level.

\section{NUMERICAL SIMULATIONS}

Numerical simulation has been used as a useful tool for evaluating the liquefaction resistance of improved grounds (e.g., Zienkiewicz et al. 1999). Advantages of numerical simulation are to be able to consider various improved soil properties, such as stiffness, strength, permeability, on cyclic behavior of soils. In this study, the undrained cyclic behavior of the desaturated silica sand was simulated using LIQCA2D17. LIQCA2D17 is developed by LIQCARI and can take into account for $\mathrm{S}_{\mathrm{r}}$ effects on stiffness and quasi-overconsolidation surface in elemental simulations. 
Table. 2. Input parameters

\begin{tabular}{|c|c|c|c|c|c|c|}
\hline \multirow{2}{*}{\multicolumn{2}{|c|}{ Parameter }} & \multirow[b]{2}{*}{ Failure criterion } & \multicolumn{2}{|c|}{$\begin{array}{c}\text { Saturated } \\
\left(D_{r}=60 \%, S_{r}=100 \%\right)\end{array}$} & \multicolumn{2}{|c|}{$\begin{array}{c}\text { Desaturated } \\
\left(D_{r}=60, S_{r}=91.3 \%\right)\end{array}$} \\
\hline & & & $\begin{array}{l}\text { Modified } \\
\text { Von Mises } \\
\end{array}$ & Yasufuku & $\begin{array}{l}\text { Modified } \\
\text { Von Mises } \\
\end{array}$ & Yasufuku \\
\hline \multicolumn{2}{|l|}{ Initial void ratio } & $e_{0}$ & \multicolumn{4}{|c|}{0.756} \\
\hline \multicolumn{2}{|l|}{ Compression index } & $\lambda$ & \multicolumn{4}{|c|}{0.0090} \\
\hline \multicolumn{2}{|c|}{ Expansion index } & $\kappa$ & \multicolumn{4}{|c|}{0.0012} \\
\hline \multicolumn{2}{|c|}{$\begin{array}{l}\text { Normalized initial shear } \\
\text { modulus }\end{array}$} & $G_{0} / \sigma_{m 0}^{\prime}$ & \multicolumn{4}{|c|}{815} \\
\hline \multicolumn{2}{|c|}{$\begin{array}{l}\text { Quasi overconsolidation } \\
\text { ratio }\end{array}$} & $\mathrm{OCR}^{*}$ & \multicolumn{4}{|c|}{1.00} \\
\hline \multicolumn{2}{|c|}{ Phase transformation ratio } & $M_{m}^{*}$ & 0.856 & 0.916 & 0.856 & 0.916 \\
\hline \multicolumn{2}{|c|}{ Failure stress ratio } & $M_{f}^{*}$ & 1.313 & 1.521 & 1.313 & 1.521 \\
\hline \multicolumn{2}{|c|}{$\begin{array}{l}\text { Fraction of phase } \\
\text { transformation ratio }\end{array}$} & $\omega_{m}^{*}=M_{m e}^{*} / M_{m c}^{*}$ & - & 0.856 & - & 0.856 \\
\hline \multicolumn{2}{|c|}{$\begin{array}{l}\text { Fraction of phase failure } \\
\text { stress ratio }\end{array}$} & $\omega_{f}^{*}=M_{f e}^{*} M_{f e}^{*}$ & - & 0.726 & - & 0.726 \\
\hline \multirow{3}{*}{\multicolumn{2}{|c|}{ Hardening parameter }} & $B_{0}$ & \multicolumn{4}{|c|}{1,550} \\
\hline & & $B_{1}$ & \multicolumn{4}{|c|}{30} \\
\hline & & $C_{f}$ & \multicolumn{4}{|c|}{-} \\
\hline \multicolumn{2}{|c|}{ Anisotropy parameter } & $C_{d}$ & \multicolumn{4}{|c|}{2,000} \\
\hline \multirow{2}{*}{\multicolumn{2}{|c|}{ Dilatancy parameter }} & $D^{*}{ }_{0}$ & \multicolumn{4}{|c|}{1.0} \\
\hline & & $n$ & \multicolumn{4}{|c|}{4.0} \\
\hline \multirow{2}{*}{ Reference strain } & Plastic & $\gamma^{P^{*}} r$ & \multicolumn{4}{|c|}{0.0022} \\
\hline & Elastic & $\gamma_{r}^{E^{*}}$ & \multicolumn{4}{|c|}{0.0080} \\
\hline \multirow{2}{*}{\multicolumn{2}{|c|}{ Suction parameter }} & $S_{I}$ & & & & \\
\hline & & $S_{I B}$ & & & & \\
\hline
\end{tabular}

\subsection{Parameter calibrations}

Table. 2 provided input parameters used for the numerical simulation. The parameters were calibrated by fitting to the experimental results. The desaturation effects should be reasonably accounted. The calibration process was briefly described herein.

A shear modulus, $G$, is an important soil property which links stresses to strains. An elastic shear modulus of soils is usually estimated by measuring shear wave velocities, $V_{s}$. An elastic shear modulus of soils is usually estimated by measuring shear wave velocities, $V_{s}$. Several laboratory studies show that $S_{r}$ has influences on $V_{s}$ at low to high degrees of saturation for cohesionless soils (e.g., Cho and Santamarina 2001). Based on such experimental results, the value of $V_{s}$ for desaturated conditions increased to be consistent with the experimental facts. For this study, no shear wave velocities were measured, so that an initial value of shear moduli, $G^{E P}$, was determined for saturated conditions as referring to the Toyoura sand's value measured by Toki et al. (1995). The value of $G^{E P}$ for desaturated conditions was determined by modifying a suction parameter, $S_{I B}$, with fitting modulus reduction behavior shown in Fig.5.

As shown in Fig.3, the experimental results showed that the angle of a critical state line, $\phi_{c l}$, was mostly the same in compression and extension regardless of a degree of saturation, $S_{r}$, and the angle of a phase transformation line, $\phi_{p l}$, for the desaturated condition increased in $4-6^{\circ}$ in extension compared to the saturated sands. Therefore, there was a need of adjustment of $\phi_{p l}$ with $S_{r}$ for the simulation. For this study, the modified Von Mises failure criterion (MVM criterion) and the Yasufuku failure criterion (YF criterion) were used. The YF criterion can take into account for a fraction of the stress ratio at a phase transformation and critical state in triaxial compression and extension. The phase transformation ratio, $M_{m}^{*}$, and the failure stress ratio, $M_{f}^{*}$, were calculated using the experimental results. For the YF criterion, the relatively large $M_{m}^{*}$ and $M_{f}^{*}$ were used compared to the MVM criterion.

Compression index $\lambda$ and expansion index $\kappa$ control volumetric strain in normally and over consolidated condition, respectively. In the author's knowledge, $S_{r}$ effects on both $\lambda$ and $\kappa$ of cohesionless soils at a high $S_{r}$ is merely investigated. There is no cohesion on the determination of these parameters. Then, the same value was used regardless of $S_{r}$. The parameters of $\lambda$ and $\kappa$ were selected referring Toyoura sand's value (e.g., Pradhan et al. 1989).

Hardening parameters, $B_{0}$, and $B_{l}$, dilatancy parameters, $D^{*}{ }_{0}$, and $n$ and reference strains, $\gamma^{P *}{ }_{r}$, and 

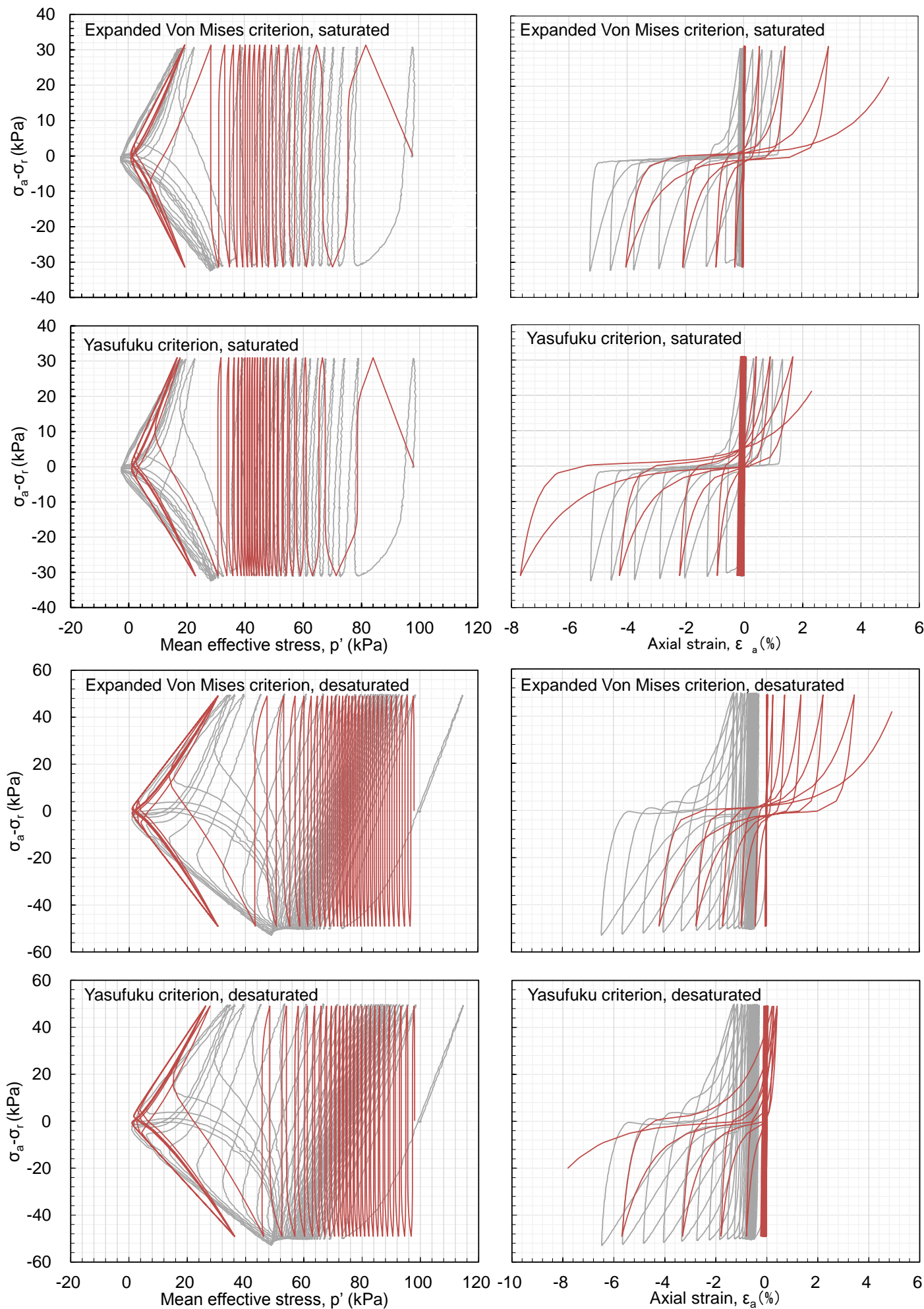

Fig. 6. Comparisons of numerical simulation to experimental results. The red line is the numerical simulation results, and the gray line is the experimental results.

$\gamma^{E^{*}}{ }_{r}$ were calibrated through trial and error. The axial strain-time history, the excess pore water pressure-time history, and the axial stress-axial strain relationships obtained from the experiments were compared to the numerically simulated those ones. Additionally, the liquefaction resistance curves shown in Fig.4 were targeted as well for the calibration. Because there is no cohesion to determine an anisotropy parameter, $C_{d}$, the same value recommended in the LIQCA2DI7 manual (LICALI 2017) was used. Once the parameters for the saturated condition were determined, the suction parameters, $S_{I}$ and $S_{I B}$ were calibrated by fitting to the experimental results of the desaturated condition.

LIQCA2D17 equips four modulus reduction 
methods after stress path reaching phase transformation. For this study, the modulus reduction methods recommended by Oka et al. (1999) was used for both the MVM criterion and the YF criterion.

\subsection{Numerical analysis results and Discussions}

Fig. 6 compared the stress path in p'-q space and the stress-strain relationship for both saturated and desaturated sands. The red line is the numerical simulation results, and the gray line is the experimental results. The experimental results shown in Fig.1 were simulated, so the parameters provided in Table 2 was calibrated for the condition of $S_{r}=91.3 \%$ and $D_{r}=60.7 \%$.

For the saturated condition, both the MVM criterion and the YF criterion developed similar stress paths. In the stress-strain relationships, the MVM criterion developed the axial strain, $\varepsilon_{a}$, symmetrically, and the YF criterion induced large axial strains in extension. The comparisons with the experimental results showed that the YF criterion simulated similar stress-strain relationships under triaxial stress condition. For the desaturated condition, the experimental results showed the small increments of the EPWP for the $N_{c y c} \approx 0-20$ and drew a large "butterfly" in the stress path for the $N_{c y c} \approx 20-30$ after the phase transformation. On the other hand, the simulation resulted in the different stress path from the experimental results. The mean effective stress started to decrease at the beginning of loading, and the stress path was still similar to the saturated condition during unloading and reloading after the phase transformation. That showed that the analysis did not simulate pore water pressure response robustly. Moreover, the failure criteria significantly influenced stress-strain relationships. The MVM criterion developed the relationships symmetrically as like the saturated condition parameter, however, the YF criterion developed large axial strains in extension. As shown in the experimental results, the axial strain, $\varepsilon_{a}$, was developed in extension, so that the YF criterion could simulate similar stress-strain loops comparing to the MVM criterion as same as the saturated condition parameters.

Fig.4 compared the liquefaction resistance curves of the numerical simulation to the experimental results based on double amplitude axial strain of the DA excess in $5 \%$. Both criteria estimated mostly the same liquefaction resistance curves. The computed curves for the saturated condition were fitted well to the experimental results. For the desaturated condition, a slope of the curves was relatively large, and the liquefaction resistance was slightly overestimated for both criteria at the small number of cyclic stress. The slope of the curves became small at a large number of cycles, and the estimated resistances matched well with the experimental results. As stated above, only the differences for the simulation between saturated and desaturated conditions were whether considering suction parameters or not. So, the use of well-calibrated parameters for the saturated condition is critical for desaturated sand simulations.

Fig.5 (A) and (B) compared the modulus reduction curves and the damping ratio, $h$, of the numerical simulations to the experimental results. For the desaturated condition, the $E_{e q} / \sigma^{\prime}{ }_{\mathrm{c} 0}$ at the initial axial strain (say, $\varepsilon_{a},=0.0001 \%$ ) was higher than the saturated condition. The $E_{e q} / \sigma^{\prime}{ }_{\mathrm{c} 0}$ for the saturated condition started to decrease around the $\varepsilon_{a}=0.001 \%$, however, the one of the desaturated condition did not change until the $\varepsilon_{a}$ reached around $0.01 \%$. The $E_{e q} / \sigma^{\prime}{ }_{\mathrm{c} 0}$ more slowly decreased than the saturated condition, which was consistent with the experimental results. The computed damping ratio was mostly the same at the small axial strains $\left(\varepsilon_{a}=0.0001-0.1 \%\right)$ for both conditions, and that ratio of the desaturated condition at $\varepsilon_{a}=0.1-1.0 \%$ was slightly higher than the saturated condition. Peak damping ratio was smaller than the experimental results. Those were contrary to the experiment results. The numerical simulation results showed that modulus reduction behavior was simulated well; however, the damping ratio was not predicted robustly.

The numerical simulation results were summarized below.

1) The modulus reduction behavior was reasonably simulated for both the saturated and desaturated conditions; however, the estimated damping ratio was contrary to the experimental results. There is a need for careful considerations for damping ratio estimations.

2) Failure criteria significantly influenced stress-strain relationships. The YF criterion was suitable for simulating both saturated and desaturated sand under triaxial stress conditions.

\section{DISCUSSIONS}

The experimental results showed that the stress-strain relationships of the desaturated condition differed from the saturated one, specifically after the stress path reached the phase transformation. Fig.7 showed relationships between accumulated axial strains from stress reverse points, $\varepsilon_{a}^{P}$,-normalized plastic young modulus, $E^{P} / \sigma_{m 0}$. Colored lines showed the relationships before phase transformation, and solid lines showed after phase transformation, respectively. For the saturated condition, the $E^{P} / \sigma^{\prime}{ }_{m 0}$ decreased with an increase of the $N_{c y c}$ and the minimum value of the $E^{P} / \sigma^{\prime}{ }_{m 0}$ was observed at the $N_{c y c}$ of $17.0-17.5$. The stiffness was rapidly reduced after the stress path reached phase transformation, and liquefaction of the saturated silica sand was characterized as "flow deformation" which is usually observed for clean sands. For the desaturated condition, the $E^{P} / \sigma_{m 0}^{\prime}$ decreased with an increase of the $N_{c y c}$ as well, however, the $E^{P} / \sigma_{m 0}^{\prime}$ continuously decreased after the stress path reached the phase transformation. The desaturated 

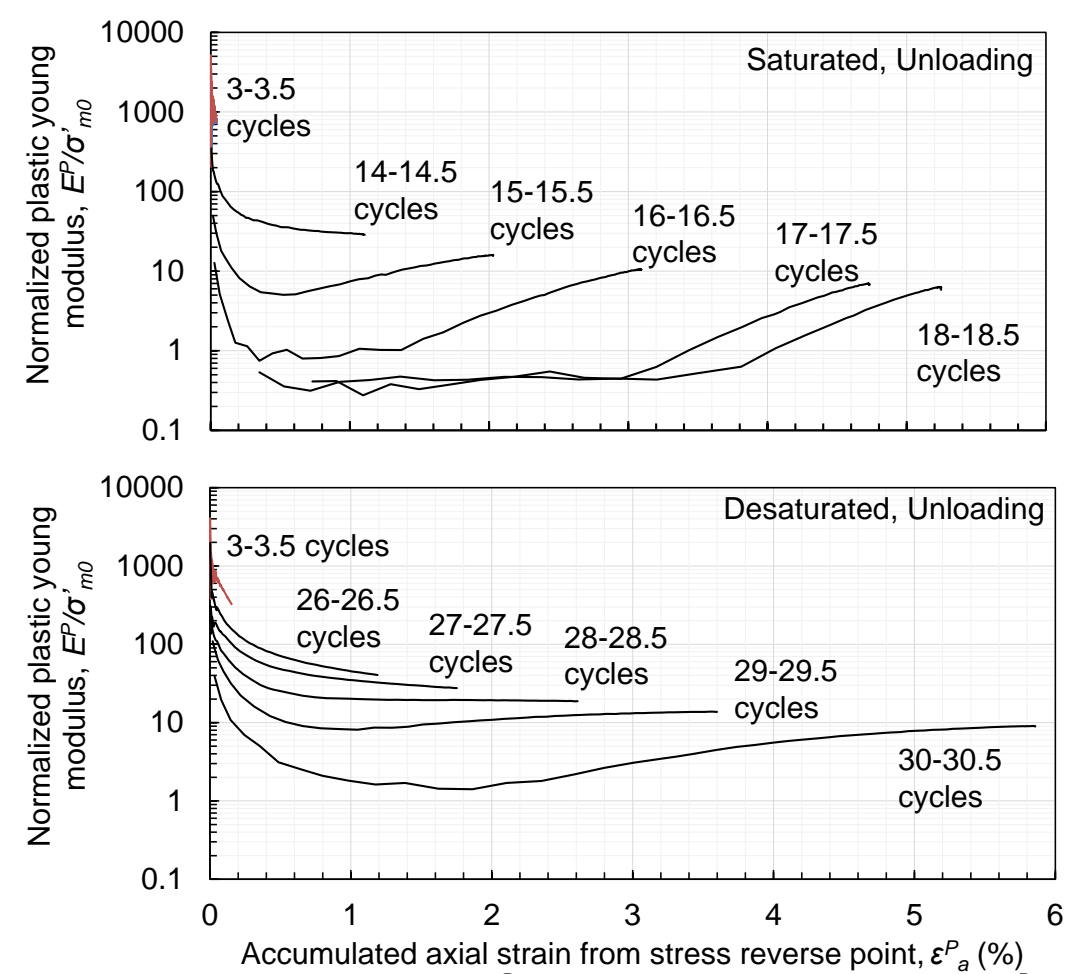

Fig. 7. Accumulated axial strain from reverse point, $\varepsilon_{a}^{P}$ - normalized plastic young modulus, $E^{P} / \sigma^{\prime}{ }_{m 0}$, relationships.
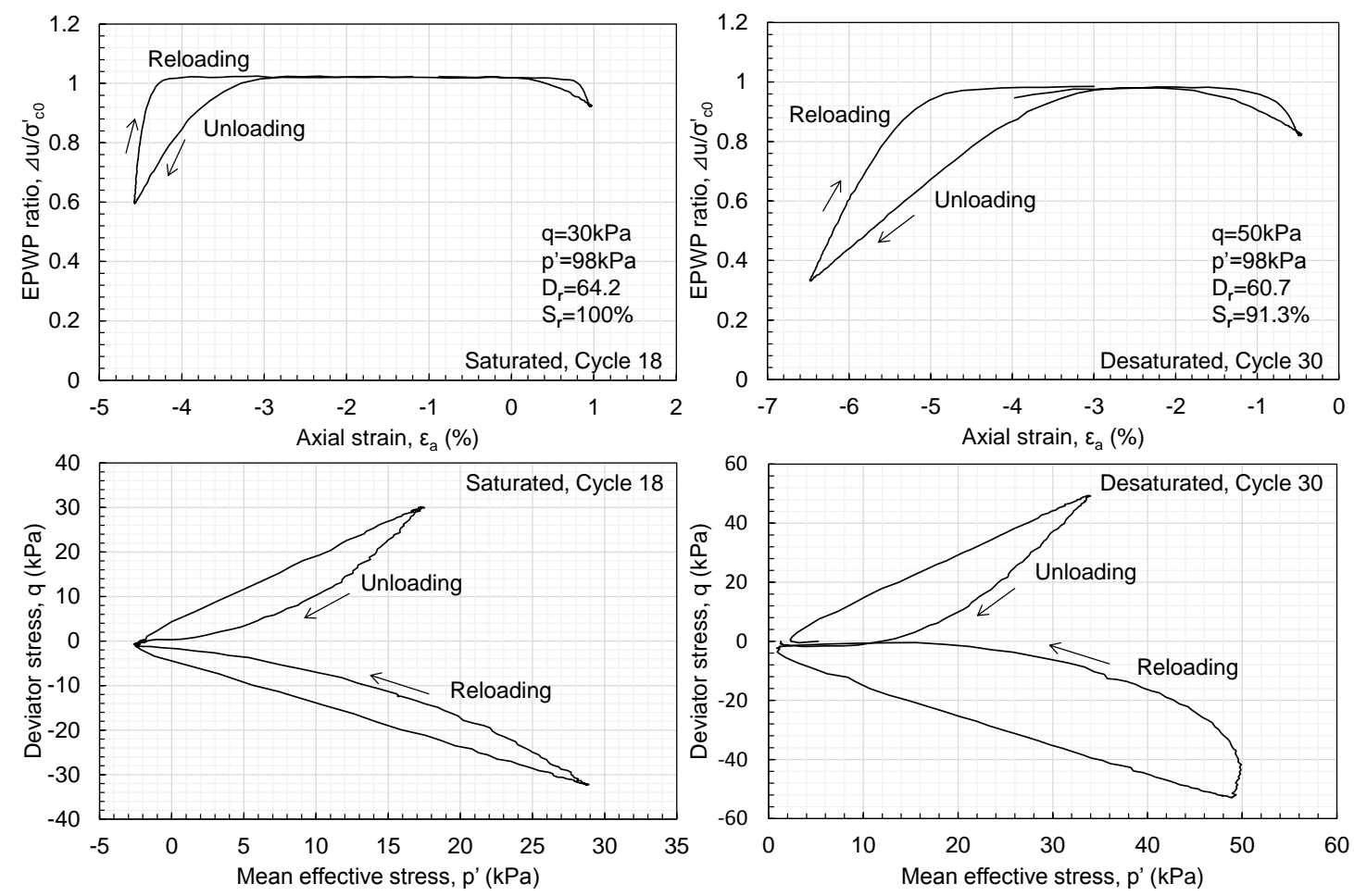

Fig. 8. EPWP ratio-axial strain, $\varepsilon_{a}$, relationships and the stress-strain relationships path of the cycle for the DA in excess $5 \%$.

sands had a relatively high deformation resistance compared to the saturated condition. So that, liquefaction of the desaturated silica sand was characterized as "slowly flow deformation."

In this study, the desaturated specimens with the high $S_{r}(=91-94 \%)$ was examined. As shown in Fig.1, the desaturated specimens reached the mean effective stress nearly zero during unloading and reloading in liquefaction. That means that the confining stress was also significantly reduced so that frictional forces acting between particles became very small. However, Fig. 8 showed that modulus reduction still decreased with an 
increase of the $N_{c y c}$ during liquefaction. Although the EPWP ratio was the same degree as the saturated specimens, the deformation characteristic was influenced by $S_{r}$. Fig.8 replotted the $\Delta u / \sigma^{\prime}{ }_{m 0}-\varepsilon_{a}$ relationships and the stress-strain curves of the cycle for the DA in excess of 5\%. For the saturated condition, the EPWP ratio immediately increased when reloading started, and the $\varepsilon_{a}$ rapidly increased when the EPWP ratio nearly equaled 1.0. For the desaturated condition, the EPWP ratio gradually increased with an increase of the $\varepsilon_{a}$ under reloading relative to the saturated one, and the $\varepsilon_{a}$ quickly increased when the EPWP ratio nearly equaled 1.0. So, the EPWP increment rate under the $\varepsilon_{a}$ increments was slower than the saturated condition. Additionally, for the saturated sands, the $p$ ' immediately decreased when the reloading began; however, for the desaturated condition, the $p$ ' was mostly constant at the beginning of the reloading and largely decreased around stress reverse (i.e., $q \approx 0 \mathrm{kPa}$ ). That indicated that $p^{\prime}$ reduction rate (i.e., EPWP increment rate) was slower than the saturated condition, which may result in relatively high young modulus during "butterfly." Therefore, the comparisons indicate that the EPWP increment rate critically influences the stress-strain relationships. Okamura and Noguchi (2009) discussed the effects of $S_{r}$, on undrained cyclic resistance of unsaturated silts. They indicated that apparent bulk modulus reductions of pore fluid influenced liquefaction resistance at low matric suction. So, the desaturation effects on liquefaction resistance should be accounted for pore fluid compressibility and volume change characteristics. Therefore, carefully calibrated parameters, specifically relating to pore fluid compressibility and volume change characteristics, can provide reasonable results for desaturated sands in elemental simulations.

\section{CONCLUSIONS}

A series of the experiments were conducted to investigate the desaturation effect on the undrained triaxial cyclic behavior of the silica sand. Desaturation was achieved by injecting micro-bubbled water. The experimental results under $D_{r}=60-65 \%$ and $S_{r}=91-100 \%$ conditions showed that the $\phi_{c l}$ was mostly the same regardless of $S_{r}$, however, the $\phi_{p l}$ increased in $4-5^{\circ}$ in extension for the desaturation condition. Additionally, the young modulus was less degraded, and the damping ratio was reduced compared to the saturated condition.

The numerical analysis was performed using LIQCA2D17 to simulate the experimental results. The comparisons to the experimental results indicated that the failure criteria significantly influenced stress-strain behavior, and the YF criterion was suitable to simulate the desaturation condition. The liquefaction resistance curves were predicted well comparing to the experimental result. The $E_{e q} / \sigma^{\prime} 0$ was also well predicted for both saturated and desaturated conditions. However, the damping ratio was not predicted robustly.

The effect of desaturation on stress-strain relationships was further discussed. The desaturation effects were critical after the stress path reached the phase transformation and the excess pore water pressure ratio equaled to 1.0. The saturated sands immediately lost the stiffness after the phase transformation in few cycles and transitioned to flow deformation. However, the desaturated sands exhibited the continuous modulus reduction although the specimens were fully liquefied. Therefore, liquefaction of those desaturated sands was characterized as "slowly flow deformation."

\section{ACKNOWLEDGMENTS}

The authors thank Dr. Azegami who conducted the undrained cyclic triaxial tests for both saturated and desaturated silica sands. The experimental data was very useful to validate the numerical simulation for desaturated conditions.

\section{REFERENCES}

1) Azegami, Y. (2008): Development of desaturation method for liquefaction mitigation using micro-bubbled water. M.S. thesis, Urban and Civil Engineering, Tokyo City University (In Japanese).

2) Boulanger, R.W., and Idriss, I.M. (2014). : CPT and SPT based liquefaction triggering procedures. Report No. $U C D / C G M-14 / 01$. Center for Geotechnical Modeling, Department of Civil and Environmental Engineering, University of California, Davis, CA, 134.

3) Cho, G. C., and Santamarina, J. C. (2001). : Unsaturated particulate materials - particle level studies, ASCE Geotechnical Journal, 127, no. 1.

4) LIQCARI (LIQCA Liquefaction Geo-Research Institute). (2017). : LIQCA2D17. LIQCA3D17 manual.

5) Marasini, N., Okamura, M., (2015). Air injection to mitigate liquefaction under light structures. Int. J. Phys. Model. Geotech. 15 (3), 129-140.

6) Oka, F., Yashima, A., Tateishi, A., Taguchi, Y. and Yamashita, S. (1999): A cyclic elastoplastic constitutive model for sand considering a plastic-strain dependence of the shear modulus, Geotechnique, 49(5), 661-680.

7) Okamura, M., Noguchi, K., (2009). : Liquefaction resistance of unsaturated nonplastic silt. Soils and Found. 49, No. 2, 221-229.

8) Okamura, M. and Soga, Y. (2006): Effects of pore fluid compressibility on liquefaction resistance of partially saturated sand, Soils and Found, 46, No.5, 93-104.

9) Toki, S., Shibuya, S., and Yamashita, S. (1995). : Standardization of laboratory test methods to determine the cyclic deformation properties of geomaterials in Japan. International symposium, Pre-failure deformation of geomaterials, Balkema, Vol.2, pp 741-784.

10) Tsukamoto, Y., Ishihara, K., Nakazawa, H., Kamada, K., and Huang, Y. (2002). : Resistance of partly saturated sand to liquefaction with reference to longitudinal and shear wave velocities. Soils and Found. 42, No.6, 93-104.

11) Yoshimi, Y., Tanaka, K. \& Tokimatsu, K. (1989). 
Liquefaction resistance of a partially saturated sand. Soils and Found. 29, No. 3, 157-162.

12) Zienkiewicz, OC., Chan AHC., Pastor M., Shrefler BA., and Shiomi T. (1999). : Computational Geomechanics. Wiley: New York. 